\title{
Analysis of the correlation between serum resistin and the variability of erythropoietin responsiveness in patients with chronic kidney disease
}

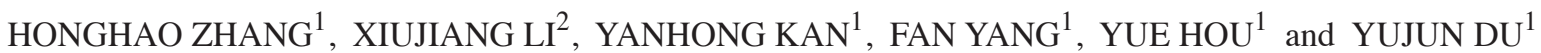 \\ ${ }^{1}$ Department of Nephrology, The First Hospital of Jilin University, Changchun, Jilin 130021; ${ }^{2}$ Intensive Care Unit, \\ Tumor Hospital of Jilin, Changchun, Jilin 130012, P.R. China
}

Received October 25, 2013; Accepted February 5, 2014

DOI: $10.3892 / \mathrm{etm} .2015 .2772$

\begin{abstract}
Chronic kidney disease (CKD) is commonly accompanied by inflammation and anemia; however, the pathogenesis of CKD is unclear. Expression of resistin, a cysteine-rich secretory plasma protein, is correlated with the expression of tumor necrosis factor (TNF)- $\alpha$, interleukin (IL)-6 and lipoprotein-associated phospholipase A2, indicating that resistin may be involved in inflammatory events. In addition, inflammation inhibits the activity of erythropoietin (EPO) and, thus, erythropoiesis. The aim of the present study was to analyze the correlation between serum resistin and the variability of EPO responsiveness in CKD patients. The levels of serum creatinine ( $\mathrm{SCr}$ ), C-reactive protein (CRP), total cholesterol, triglycerides, IL-6 and serum resistin were measured in the samples obtained from 138 CKD patients and healthy control subjects. The levels of serum resistin in the CKD groups with and without hemodialysis were significantly higher than those observed in the normal control group $(\mathrm{P}<0.01)$ and the levels of serum resistin in the hemodialysis CKD group were higher than those observed in the CKD group without dialysis $(\mathrm{P}<0.01)$. The levels of serum resistin in patients in the randomly selected CKD group (with hemodialysis) were positively correlated with the duration of dialysis and the levels of $\mathrm{SCr}$ and CRP $(\mathrm{P}<0.05)$, however, were negatively correlated with the estimated glomerular filtration rate. The EPO resistance index (ERI) was identified to be associated with body mass index and the levels of CRP and resistin; furthermore, EPO reactivity was correlated with the level of resistin and ERI. The levels of serum resistin were correlated with the vari-
\end{abstract}

Correspondence to: Dr Yujun Du, Department of Nephrology, The First Hospital of Jilin University, 71 XinMin Street, Changchun, Jilin 130021, P.R. China

E-mail:kjkduyujun@126.com

Key words: chronic kidney disease, resistin, inflammation, anemia, erythropoietin, erythropoietin resistance index ability in EPO responsiveness that was observed in the CKD patients. Therefore, the measurement of serum resistin may aid with understanding the mechanisms, clinical diagnosis and treatment of CKD.

\section{Introduction}

Chronic kidney disease (CKD), a worldwide problem with a high incidence, is commonly accompanied by inflammation and anemia. Between 6.5 and $10 \%$ of the population in developed countries suffer from various renal diseases (1). CKD is a condition, which is characterized by chronic inflammation and is hypothesized to be promoted by cytokines and oxidation reactions. The inflammation can be induced by toxins, immune complexes, microorganisms, chemical agents and complement, and is activated by the monocyte-macrophage system during which tumor necrosis factor (TNF)- $\alpha$, interleukin (IL)- 6 and other agents are released (1). However, the underlying pathogenesis of the numerous forms of CKD remains unclear.

Expression of resistin, a cysteine-rich secretory plasma protein (molecular weight, $\sim 12.5 \mathrm{kDa}$ ), is correlated with the expression of TNF- $\alpha$, IL- 6 , lipoprotein-associated phospholipase A2 and other factors, which indicates that it may be involved in various inflammatory events. A number of studies have identified that resistin was involved in insulin resistance and metabolic syndromes in renal diseases (1-3) and CKD patients on maintenance hemodialysis often succumbed to cardiovascular and cerebrovascular complications (4). Elevation of resistin levels may serve as a marker of atherosclerosis (AS) in patients exhibiting a metabolic syndrome, indicating that resistin may be key in the occurrence and development of coronary AS (1-3) as well as demonstrating that there may be a correlation between resistin levels and heart disease in CKD patients.

Anemia often occurs in CKD patients and is closely associated with a high incidence of cardiovascular disease. Recombinant human erythropoietin (EPO) has been administered to CKD patients presenting with anemia in addition to undergoing hemodialysis. However, there is evidence that a high dose of EPO may be associated with an increased rate of mortality. Boyraz et al (2) demonstrated that administration of a high dose of EPO to treat anemia may lead to an increase 
in mortality. Chen et al (3) reported that EPO responses and targeted hemoglobin $(\mathrm{Hb})$ values may serve as markers of mortality risk. Therefore, prescribing an excessive dose of EPO for CKD patients may increase mortality. Moreover, EPO was involved in inflammation (2) and the inflammation inhibited the activity of EPO and, thus, erythropoiesis of CKD patients, which indicated that there may be a correlation between inflammation and EPO responsiveness in CKD patients. As changes in the levels of EPO and resistin may be involved in the occurrence and progression of CKD, the correlation between serum resistin and variability to EPO responsiveness, in tissue samples obtained from CKD patients, was investigated in the present study.

\section{Materials and methods}

Materials. An enzyme-linked immunosorbent assay (ELISA) kit for detecting human resistin was obtained from Quantikine (Minneapolis, MN, USA) and IL-6 was purchased from Invitrogen Life Technologies (Carlsbad, CA, USA). Recombinant EPO was purchased from Life Sciences Advanced Technologies (St. Petersburg, FL, USA) and additional reagents were purchased from Sigma-Aldrich (St. Louis, MO, USA).

Patients. The CKD patients $(\mathrm{n}=138)$ and healthy control subjects $(n=16)$ were recruited from the First Hospital Affiliated to Bethune Medical College of Jilin University (Changchun, China) between May 2009 and May 2012. Ethical approval was obtained from the institutional review committee of Jilin University (Changchun, China) and informed consent was obtained from the participants according to the Declaration of Helsinki (4). The samples were analyzed by routine blood tests to determine coagulation, liver and renal function, as well as the levels of blood sugar, blood lipids (high density lipoproteins and low density lipoproteins), ions and parathyroid hormone. In addition, gender, age, dialysis duration, systolic and diastolic blood pressure and cardiac functional grades were recorded.

Patient grouping. One hundred and thirty-eight CKD patients were divided into two groups; one group did not receive hemodialysis and the second group did. The healthy subjects served as a control group and the patients in the second group (the hemodialysis group), were randomly selected. The patients of the third group, a subset of the second group, were subjected to hemodialysis for $>3$ months in addition to intravenous EPO therapy to maintain a $\mathrm{Hb}$ level of $11.0 \mathrm{~g} / \mathrm{l}$.

The CKD groups without dialysis were designated as CKD3, 4 and 5. The CKD3 group comprised of six males and five females (mean age, 39.8 44.9 years), the CKD4 group comprised of six males and six females (mean age, $48.0 \pm 4.9$ years) and the CKD3 group comprised of eight males and six females (mean age, $38.9 \pm 17.5$ years).

The CKD patients, who received dialysis, were divided into two groups as described above; the first group included 21 males and 18 females (mean age, $47.5 \pm 23.8$ years) and the second group included 33 males and 29 females (mean age, $53.5 \pm 21.9$ years) with a dialysis time of $39.8 \pm 15.7$ months The first group was dialyzed three times per week for four hours each time using a 4008s dialysis machine (Fresenius Medical Care, Waltham, MA, USA). Blood flow was measured at $200-220 \mathrm{ml} / \mathrm{min}$ using bicarbonate as the dialysate and a dialysis rate of $500 \mathrm{ml} / \mathrm{min}$.

The patients in the second CKD group, who underwent dialysis, received dialysis and treatment for $>3$ months and were injected with EPO to maintain Hb levels of $11.0 \mathrm{~g} / \mathrm{l}$. The patients that were receiving dialysis were also administered with ferrous sulfate and oral folic acid to maintain serum ferritin levels at $>100 \mathrm{ng} / \mathrm{ml}$ and transferrin saturation (TSAT) at $>20 \%$.

The CKD patients, who received hemodialysis, were administered with ferrous sulfate and folate to maintain serum ferritin levels at $>100 \mathrm{ng} / \mathrm{ml}$ and TSAT at $>20 \%$. When the serum ferritin levels were $<100 \mathrm{ng} / \mathrm{ml}$ and TSAT $<20 \%$, the patients who were subjected to 10 continuous cycles of hemodialysis were administered with $100 \mathrm{mg}$ iron hydroxide or were removed from the study (1-3). In addition, when serum ferritin levels were $\geq 800 \mathrm{ng} / \mathrm{ml}$ and TSAT $\geq 50 \%$, the patients were excluded from the study.

The healthy control group included nine males and seven females (mean age, 37.6 \pm 10.5 years).

Blood preparation. Blood was drawn from a vein in the arm and mixed with sodium citrate, which served as an anticoagulant. The blood was sent to the clinical laboratory from the First Hospital Affiliated to Bethune Medical College of Jilin University for further experiments. The samples were centrifuged at $460 \mathrm{x}$ g (Allegra X-15 R centrifuge, Beckman Coulter, Brea, CA, USA) for $8 \mathrm{~min}$ at room temperature and the supernatant was obtained and subjected to determine the levels of serum creatinine ( $\mathrm{SCr}$ ), blood urea nitrogen and serum albumin.

ELISA. All of the samples were subjected to routine blood analysis. The levels of $\mathrm{SCr}$, serum $\mathrm{C}$-reactive protein (CRP), serum total cholesterol, triglycerides, IL-6 and serum resistin were detected in all of the groups using sandwich ELISA. ELISA assays were performed as previously reported (5). Briefly, $100 \mu \mathrm{l}$ diluent was added to each well of an RD1-19 plate (Sigma, St. Louis, MO, USA) in addition to $100 \mu \mathrm{l}$ standard solution, control solution and sample. The wells were blocked using a rubber sealing bar and the mixture was incubated for $2 \mathrm{~h}$ at room temperature. Each well was washed three times with four repetitions each time and $200 \mu \mathrm{l}$ resistin conjugating agent was added. The wells were blocked an additional time, using a rubber-sealing bar and incubated for $2 \mathrm{~h}$ at room temperature. Subsequent to washing each well again, a substrate solution was added and the reactions were conducted in the dark for $30 \mathrm{~min}$ at room temperature. Stopping buffer $(50 \mu \mathrm{l})$ was added and the solutions were gently mixed, the change in the color of the solution in each well from blue to yellow, indicated that the reaction was finished. The light intensity of each well was measured after 30 min using an ELISA reader (Sigma) with an absorbance of $450 \mathrm{~nm}$.

The measurement of CRP was conducted using a fully-automated DADE Behring BN ProSpec (Beckman Coulter) using ancillary reagents. Immunosuppressant rate nephelometry was used according to the manufacturer's instructions. 
Table I. Clinical data of study participants.

\begin{tabular}{|c|c|c|c|c|c|c|}
\hline \multirow[b]{2}{*}{ Variable } & \multicolumn{3}{|c|}{ CKD group without hemodialysis } & \multicolumn{2}{|c|}{ Hemodialysis CKD group } & \multirow{2}{*}{$\begin{array}{l}\text { Control } \\
\text { group }\end{array}$} \\
\hline & CKD3 & CKD4 & CKD5 & 1 & 2 & \\
\hline Case number & 11 & 12 & 14 & 39 & 62 & 16 \\
\hline Gender (m/f) & $6 / 5$ & $6 / 6$ & $8 / 6$ & $21 / 18$ & $33 / 29$ & $9 / 7$ \\
\hline Age (years) & $39.8 \pm 4.9$ & $48.0 \pm 4.9$ & $38.8 \pm 17.5$ & $47.5 \pm 23.8$ & $53.5 \pm 21.9$ & $37.6 \pm 10.5$ \\
\hline BMI & $25.1 \pm 3.7$ & $23.3 \pm 4.7$ & $23.5 \pm 3.4$ & $23.2 \pm 3.7$ & $23.0 \pm 4.3$ & $22.0 \pm 3.1$ \\
\hline $\begin{array}{l}\text { Dialysis time } \\
\text { (months) }\end{array}$ & - & - & - & $40.1 \pm 15.9$ & $39.8 \pm 15.7$ & - \\
\hline $\mathrm{Hb}(\mathrm{g} / \mathrm{l})$ & $128 \pm 22$ & $107 \pm 14$ & $92.7 \pm 19.4$ & $87.5 \pm 17.6$ & $104 \pm 7$ & $123 \pm 20$ \\
\hline $\mathrm{HCT}$ & $0.36 \pm 0.02$ & $0.28 \pm 0.05$ & $0.26 \pm 0.08$ & $0.21 \pm 0.09$ & $0.30 \pm 0.02$ & $0.42 \pm 0.03$ \\
\hline Alb (g/l) & $37.5 \pm 4.3^{\mathrm{a}}$ & $35.0 \pm 5.2^{\mathrm{a}}$ & $33.9 \pm 5.3^{\mathrm{a}}$ & $30.4 \pm 8.2^{\mathrm{a}}$ & $31.0 \pm 4.4^{\mathrm{a}}$ & $47.9 \pm 4.2$ \\
\hline $\mathrm{TC}(\mathrm{mmol} / \mathrm{l})$ & $4.59 \pm 1.02^{\mathrm{a}}$ & $4.88 \pm 1.10^{\mathrm{a}}$ & $5.04 \pm 1.19$ & $5.28 \pm 1.37^{\mathrm{a}}$ & $5.37 \pm 1.36$ & $4.27 \pm 1.41$ \\
\hline TG (mmol/l) & $2.29 \pm 1.79^{a}$ & $1.91 \pm 0.73^{\mathrm{a}}$ & $1.73 \pm 0.94^{\mathrm{a}}$ & $2.83 \pm 0.71^{\mathrm{a}}$ & $2.96 \pm 0.81^{\mathrm{a}}$ & $1.34 \pm 0.51$ \\
\hline HDL (mmol/l) & $1.23 \pm 0.41^{\mathrm{a}}$ & $1.09 \pm 0.34^{\mathrm{a}}$ & $0.99 \pm 0.15^{\mathrm{a}}$ & $0.75 \pm 0.09^{\mathrm{a}}$ & $0.80 \pm 0.02^{\mathrm{a}}$ & $1.42 \pm 0.63$ \\
\hline LDL (mmol/l) & $2.57 \pm 0.74^{\mathrm{a}}$ & $2.39 \pm 0.87^{\mathrm{a}}$ & $2.34 \pm 0.81^{\mathrm{a}}$ & $2.57 \pm 0.64^{\mathrm{a}}$ & $2.53 \pm 0.68^{\mathrm{a}}$ & $2.34 \pm 0.46$ \\
\hline $\mathrm{SCr}(\mu \mathrm{mol} / \mathrm{l})$ & $231 \pm 69^{a}$ & $564 \pm 96^{\mathrm{a}}$ & $1280 \pm 311^{\mathrm{a}}$ & $793 \pm 296^{a}$ & $776 \pm 258^{a}$ & $60.8 \pm 10.6$ \\
\hline CRP (mg/l) & $4.28 \pm 4.84^{\mathrm{a}}$ & $5.30 \pm 4.24^{\mathrm{a}}$ & $7.06 \pm 4.31^{\mathrm{a}}$ & $4.94 \pm 2.73^{\mathrm{ab}}$ & $5.23 \pm 3.19^{\mathrm{ab}}$ & $1.89 \pm 0.47$ \\
\hline IL-6 (ng/ml) & $2.05 \pm 1.03^{\mathrm{a}}$ & $2.21 \pm 1.37^{\mathrm{a}}$ & $3.17 \pm 1.05^{\mathrm{a}}$ & $5.61 \pm 5.13^{\mathrm{ab}}$ & $5.42 \pm 5.53^{\mathrm{ab}}$ & $1.57 \pm 0.91$ \\
\hline $\mathrm{EPO}(\mathrm{IU})$ & $1.58 \pm 0.22$ & $1.76 \pm 0.68$ & $1.83 \pm 1.12$ & $1.91 \pm 1.37$ & $1.97 \pm 1.23$ & $1.45 \pm 1.02$ \\
\hline Resistin (ng/ml) & $9.48 \pm 4.11^{\mathrm{a}}$ & $14.1 \pm 6.7^{\mathrm{a}}$ & $19.3 \pm 7.1^{\mathrm{a}}$ & $23.1 \pm 4.2^{\mathrm{ab}}$ & $22.6 \pm 5.6^{\mathrm{ab}}$ & $8.72 \pm 2.32$ \\
\hline
\end{tabular}

${ }^{\mathrm{a}} \mathrm{P}<0.01$ compared with the control group and ${ }^{\mathrm{b}} \mathrm{P}<0.01$ compared with the group without dialysis. Data are presented as the mean \pm standard deviation. CKD, chronic kidney disease; BMI, body mass index; Hb, hemaglobin; HCT, hematocrit; Alb, albumin; TC, total cholesterol; TG, triglycerides; HDL, high density lipoprotein; LDL, low density lipoprotein; SCr, serum creatinine; CRP, C-reactive protein; IL, interleukin; EPO, erythropoietin.

The level of human IL-6 was measured via an ELISA method with an ELISA kit (Invitrogen Life Technologies) according to the manufacturer's instructions.

The estimated glomerular filtration rate (eGFR) was calculated using the following revised equation, termed the modification of diet in renal disease (6): $\mathrm{eGFR}=186 \times(\mathrm{SCr})^{-1.154} \times(\text { age })^{-0.203} \times 0.742$ (for females). Where the units are: eGFR, $\mathrm{ml} / \mathrm{min} / 1.73 \mathrm{~m}^{2} ; \mathrm{SCr}, \mathrm{mg} / \mathrm{dl}$ and age, years.

The EPO resistance index (ERI) was defined as: EPO dose/Hb unit/body weight/week.

Data analysis. Data are presented as means \pm standard deviation and the statistical significance between the means was analyzed using one-way analysis of variance and the $\chi^{2}$ test and Student's t-test were performed using SPSS 16.0 software (SPSS Inc., Chicago, IL, USA). Fisher's exact test was used to analyze the correlations between the levels of resistin and variability to EPO responsiveness in the CKD patients. $\mathrm{P}<0.05$ and $\mathrm{P}<0.01$ were considered to indicate statistically significant differences.

\section{Results}

Clinical data. The clinical data and demographics of the study participants, including gender, age and length of dialysis are shown in Table I.

Levels of resistin in CKD samples. The serum resistin levels in the patients in the CKD groups who were not dialyzed, and

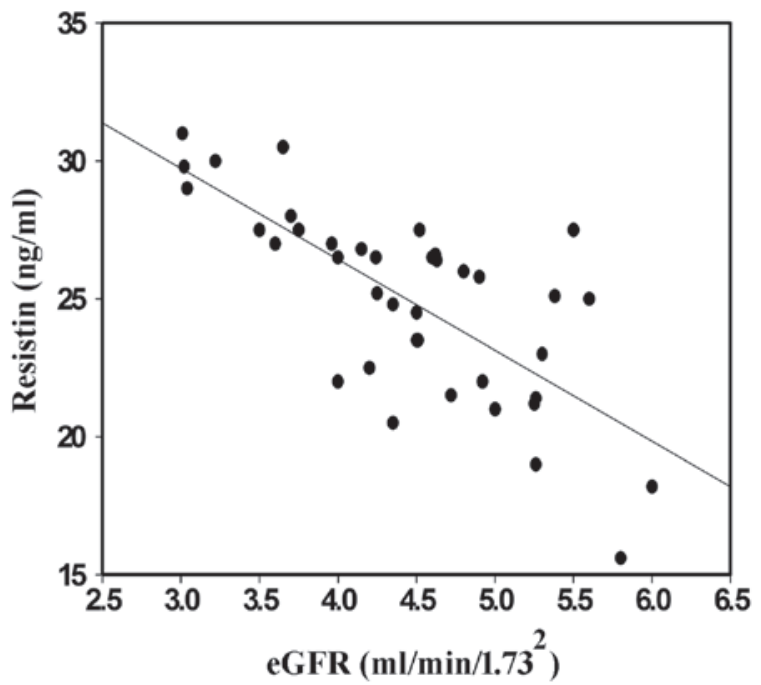

Figure 1. Scatter diagram of the correlation between serum resistin and eGFR in group 1 (with hemodialysis). The blood samples were harvested and the serum was isolated via centrifugation. The serum from group 1 was subjected to eGFR analysis by enzyme-linked immunosorbent assay and correlation analysis was performed with resistin. eGFR, estimated glomerular filtration rate.

in those in the hemodialysis CKD group were identified to be significantly higher than those in the control group $(\mathrm{P}<0.01)$. The levels of serum resistin in the hemodialysis CKD group were higher than those observed in the CKD groups without 


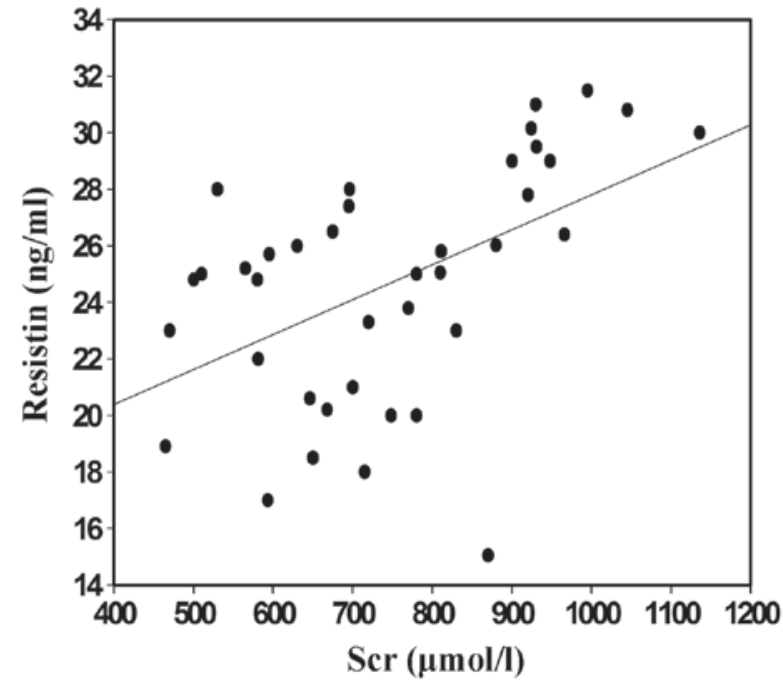

Figure 2. Scatter diagram of the correlation between serum resistin and $\mathrm{SCr}$ in group 1 (with hemodialysis). Blood samples were harvested and the serum was isolated via centrifugation (1,500 $\mathrm{x} g$ for $10 \mathrm{~min})$. The serum from group 1 was subjected to $\mathrm{SCr}$ analysis by enzyme-linked immunosorbent assay and correlation analysis was also performed with resistin. Scr, serum creatinine.

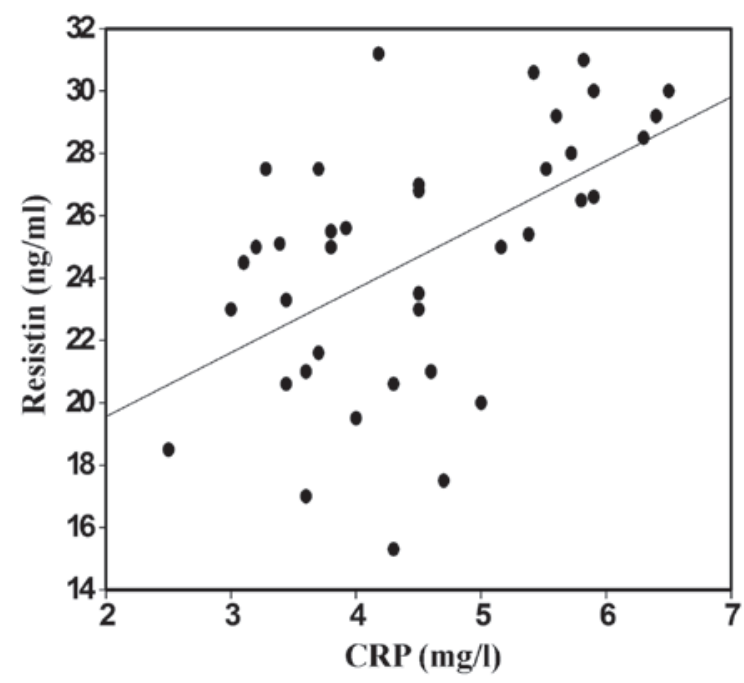

Figure 3. Scatter diagram of the correlation between serum resistin and CRP in group 1 (with hemodialysis). Blood samples were harvested and the serum was isolated via centrifugation. The serum from group 1 and the healthy control group was subjected to CRP analysis by enzyme-linked immunosorbent assay and correlation analysis was performed with resistin. CRP, C-reactive protein.

dialysis $(\mathrm{P}<0.01)$. There was a marked difference between the hemodialysis patient groups 1 and 2 with regards to level of resistin $(\mathrm{P}<0.05$; Table I).

Correlation between resistin and eGFR. The kidneys of the CKD patients and the control subjects were subjected to eGFR analysis via an enzymatic method and the correlation analysis was performed using resistin. The level of resistin increased in the CKD3, 4 and 5 groups without dialysis and statistically significant differences were identified between these three groups. However, no significant difference was identified among the groups with dialysis. These data indicated that serum resistin and eGFR were correlated (Fig. 1).

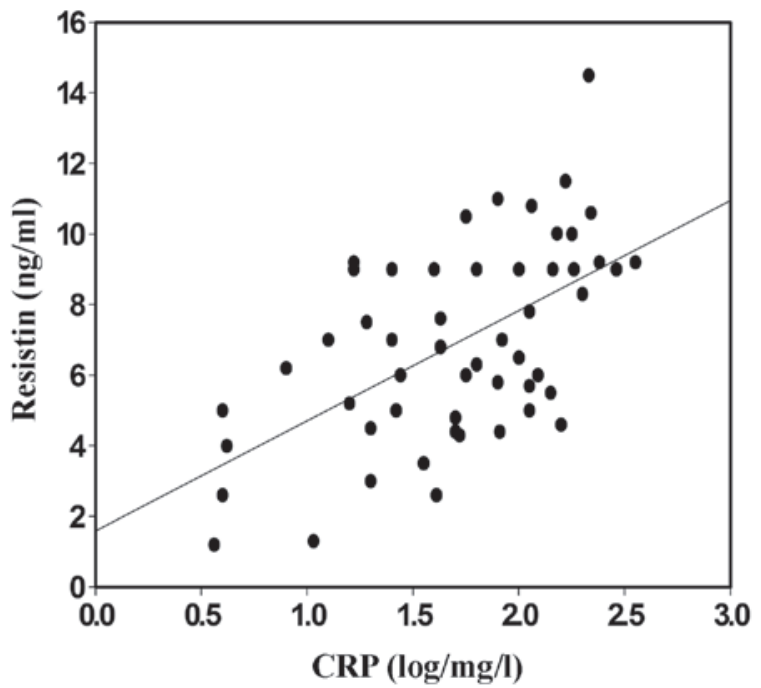

Figure 4. Scatter diagram of the correlation between serum resistin and CRP in group 2 (with hemodialysis). Blood samples were harvested and the serum was isolated via centrifugation. The serum from group 2 and the healthy control group was subjected to CRP analysis by enzyme-linked immunosorbent assay and correlation analysis was performed with resistin. CRP, C-reactive protein.

Correlation between resistin and SCr. The blood samples of the CKD patients and the control subjects were collected and subjected to ELISA analysis. The levels of serum resistin in the randomly selected CKD group with hemodialysis indicated positive correlation with the levels of $\mathrm{SCr}(\mathrm{P}<0.05$; Fig. 2).

Correlation between resistin and CRP. To investigate the clinical correlation between the highly sensitive CRP and serum resistin in the CKD patients treated with EPO, the levels of CRP were detected and compared with those of resistin. The serum levels of resistin in the randomly selected CKD group with hemodialysis were positively correlated with the levels of CRP (Figs. 3 and 4; P<0.05).

\section{Discussion}

In the present study, the data demonstrated that the serum resistin levels correlated with the variability in EPO responsiveness that was observed in CKD patients, indicating that serum resistin may serve as a marker for the occurrence and development of CKD. This conclusion is supported by numerous lines of evidence; the levels of serum resistin in CKD groups with and without hemodialysis were significantly higher than those observed in the normal control group $(\mathrm{P}<0.01)$. Furthermore, the levels of serum resistin in the hemodialysis CKD group were higher than those of the CKD group without dialysis $(\mathrm{P}<0.01)$. No marked difference was identified between the two groups of hemodialysis patients regarding the levels of resistin $(P>0.05)$. Moreover, the levels of serum resistin in the randomly selected CKD group with hemodialysis were positively correlated with the duration of the dialysis period, the levels of SCr and CRP $(\mathrm{P}<0.05)$; however, they were negatively correlated with eGFR. ERI was observed to be correlated with body mass index (BMI) and CRP and resistin levels. In addition, EPO reactivity was determined by BMI and CRP 
and resistin levels. Finally, a correlation between the level of resistin and ERI was demonstrated.

CKD, specifically in end-stage renal disease, is a condition in which chronic inflammation is present and one that is hypothesized to be promoted by cytokines and oxidation reactions. The inflammation is induced by various factors and is associated with cytokines, such as TNF- $\alpha$ and IL-6 (6). In a previous study, the levels of CRP and IL- 6 were observed to be higher in CKD patients than in the healthy control subjects, indicating that there was a micro-inflammatory state in the CKD patients (4).

Resistin, which was initially identified in obese diabetic patients, is a signaling molecule secreted by adipose cells (7). Previous studies have revealed that resistin is involved in insulin resistance and that there is a correlation between the level of plasma resistin and pro-inflammatory cytokines, whereas there is a negative correlation between the level of resistin and eGFR, in CKD patients. Patients who were on maintenance hemodialysis predominantly succumbed to cardiovascular and cerebrovascular complications (8). Elevation of the resistin level may serve as a marker of AS in patients who exhibit metabolic syndromes, indicating that resistin may be key in the occurrence and development of coronary AS. The present results supported the conclusion of Díez et al (9), who demonstrated a correlation between resistin and heart disease in patients with end-stage renal disease.

Anemia commonly occurs in CKD patients and is closely associated with a high incidence of cardiovascular disease. Recombinant human EPO has been used in hemodialysis to treat anemia patients in clinical applications and EPO is a mainstay in treating anemia in CKD patients. However, there are EPO-sensitive patients and the variability in EPO responsiveness in these patients is considerable. Moreover, although the levels of $\mathrm{Hb}$ were in the normal range, it was reported that high-dose EPO was involved in increased instances of mortality, indicating that EPO responsiveness and the targeted value of $\mathrm{Hb}$ may serve as markers for mortality risk. Therefore, it is necessary to investigate the variability of EPO responsiveness. It is possible that the administration of EPO to CKD patients may increase the risk of various diseases, and EPO and resistin may be involved in the occurrence and progression of CKD; therefore, the correlation between serum resistin and the variability of EPO responsiveness in CKD patients was investigated in the present study.

Resistin is highly expressed in normal fetal liver, adult bone, umbilical cord blood and peripheral blood cells, which indicates that the distribution of human resistin may be associated with hematopoiesis (10). In addition, granulocyte colony stimulating factor (G-CSF) induces the secretion of resistin in adipose cells in vitro and G-CSF treatment markedly increases the levels of serum resistin (11). G-CSF is critical in inhibiting erythropoiesis and anemia (12). Thus, these data demonstrated a correlation between the levels of secreted resistin and a decreased number of erythrocytes, indicating that there may be a correlation between the level of resistin and EPO responsiveness. The level of resistin was previously shown to be increased in patients exhibiting insulin resistance $(9,13)$ and in a previous study, EPO was shown to reverse insulin resistance by curing anemia in uremic patients (14). Furthermore, EPO treatment decreased the iron stores within the body to reduce the insulin resistance and improved the chronic inflammatory state in CKD patients (15).

The negative correlation between resistin and eGFR may be applied to the diagnosis of CKD patients. In the second stage of CKD $\left(e G F R=60-89 \mathrm{~mol} / \mathrm{min} / 1.73 \mathrm{~mm}^{-2}\right)(2,16-20)$, the level of resistin increased markedly, whereas other polypeptide substances were freely filtered by the kidneys. In addition, the level of resistin was observed to increase with decreasing levels of eGFR in CKD patients. In the present study, the level of resistin increased in the patients in the CKD3, 4 and 5 groups without dialysis and there was a statistically significant difference observed among these three groups. However, there was no difference identified among the two groups with dialysis. Moreover, data was obtained, which indicated that the level of resistin in CKD patients was negatively correlated with eGFR. In the present study, the level of resistin in patients with end-stage renal disease was higher than that of the healthy control subjects and the level of resistin in the groups with hemodialysis was higher than that of the renal failure groups without hemodialysis. The present results showed that resistin was positively correlated with dialysis time and $\mathrm{SCr}$, whereas, it was negatively correlated with eGFR. This indicated that resistin was associated with an inflammatory state in the patients with end-stage renal disease and was correlated with the function of the remnant kidney.

BMI has been reported to be negatively associated with the control of anemia $(19,20)$ and in the present study, EPO responsiveness was negatively correlated with BMI. However, previous studies have demonstrated that BMI was independent of the EPO response (21). Kotanko et al (22) reported that CKD patients exhibiting a high proportion of adipose tissue and subcutaneous fatty tissue required less EPO and exhibited less EPO resistance. More than 100 molecules, including cytokines that are secreted by adipose tissue, may be involved in the response to EPO. Adipose tissue participates in energy regulation, inflammation and the immune response, in addition to being a component of the hematopoietic microenvironment. In the present study, there was a high correlation observed between ERI and CRP, indicating that EPO responsiveness is involved in inflammation. Moreover, resistin is secreted by adipose tissue, thus, there may be a correlation between the levels of resistin and EPO responsiveness.

In conclusion, a correlation was observed between the levels of serum resistin and the variability of EPO responsiveness in CKD patients, which may aid in clarifying the underlying mechanism of CKD and may provide an improved method for diagnosing CKD.

\section{Acknowledgements}

The present study was supported by grants from the National Natural Science Foundation of China.

\section{References}

1. Ota T: Chemokine systems link obesity to insulin resistance. Diabetes Metab J 37: 165-172, 2013.

2. Boyraz M, Cekmez F, Karaoğlu A, Cinaz P, Durak M and Bideci A: Relationship of adipokines (adiponectin, resistin and RBP4) with metabolic syndrome components in pubertal obese children. Biomark Med 7: 423-428, 2013. 
3. Chen XY, Zhang JH, Liu F, Liu HM, Song YY and Liu YL: Association of serum resistin levels with metabolic syndrome and early atherosclerosis in obese Chinese children. J Pediatr Endocrinol Metab 26: 855-860, 2013.

4. Drouet L and Bal Dit Sollier C: Fibrinogen: factor and marker of cardiovascular risk. J Mal Vasc 27: 143-156, 2002 (In French).

5. Kim KH, Lee K, Moon YS and Sul HS: A cysteine-rich adipose tissue-specific secretory factor inhibits adipocyte differentiation. J Biol Chem 276: 11252-11256, 2001.

6. Imai E, Horio M, Nitta K, et al: Modification of the Modification of Diet in Renal Disease (MDRD) Study equation for Japan. Am J Kidney Dis 50: 927-937, 2007.

7. Steppan CM, Bailey ST, Bhat S, et al: The hormone resistin links obesity to diabetes. Nature 409: 307-312, 2001.

8. Ren W, Pan H, Wang P, et al: Clinical analysis of pulmonary infection in hemodialysis patients. Exp Ther Med 7: 1713-1717, 2014

9. Díez JJ, Iglesias P, Fernández-Reyes MJ, et al: Serum concentrations of leptin, adiponectin and resistin, and their relationship with cardiovascular disease in patients with end-stage renal disease. Clin Endocrinol (Oxf) 62: 242-249, 2005.

10. Briana DD and Malamitsi-Puchner A: The role of adipocytokines in fetal growth. Ann NY Acad Sci 1205: 82-87, 2010.

11. Lago F, Dieguez C, Gómez-Reino J and Gualillo O: The emerging role of adipokines as mediators of inflammation and immune responses. Cytokine Growth Factor Rev 18: 313-325, 2007.

12. Papaldo P, Ferretti G, Di Cosimo S, et al: Does granulocyte colony-stimulating factor worsen anemia in early breast cancer patients treated with epirubicin and cyclophosphamide? J Clin Oncol 24: 3048-3055, 2006.

13. Anderson PD, Mehta NN, Wolfe ML, et al: Innate immunity modulates adipokines in humans. J Clin Endocrinol Metab 92: 2272-2279, 2007.

14. Borissova AM, Djambazova A, Todorov K, Dakovska L, Tankova T and Kirilov G: Effect of erythropoietin on the metabolic state and peripheral insulin sensitivity in diabetic patients on haemodialysis. Nephrol Dial Transplant 8: 93, 1993.
15. Rasic-Milutinovic Z, Perunicic-Pekovic G, Cavala A, Gluvic Z, Bokan L and Stankovic S: The effect of recombinant human erythropoietin treatment on insulin resistance and inflammatory markers in non-diabetic patients on maintenance hemodialysis. Hippokratia 12: 157-161, 2008.

16. National Kidney Foundation: K/DOQI clinical practice guidelines for chronic kidney disease: evaluation, classification, and stratification. Am J Kidney Dis 39 (2 Suppl 1): S1-S266, 2002.

17. Nüsken KD, Kratzsch J, Wienholz V, Stöhr W, Rascher W and Dötsch J: Circulating resistin concentrations in children depend on renal function. Nephrol Dial Transplant 21: 107-112, 2006.

18. Ridker PM, Pare G, Parker A, et al: Loci related to metabolic-syndrome pathways including LEPR,HNF1A, IL6R, and GCKR associate with plasma C-reactive protein: the Women's Genome Health Study. Am J Hum Genet 82: 1185-1192, 2008.

19. Takeda A, Toda T, Shinohara S, Mogi Y and Matsui N: Factors contributing to higher hematocrit levels in hemodialysis patients not receiving recombinant human erythropoietin. Am J Kidney Dis 40: 104-109, 2002.

20. Di Iorio B, Cirillo M, Bellizzi V, Stellato D and De Santo NG; Campania Dialysis Registry Research Group: Prevalence and correlates of anemia and uncontrolled anemia in chronic hemodialysis patients - the Campania Dialysis Registry. Int J Artif Organs 30: 325-333, 2007.

21. Locatelli F, Andrulli S, Memoli B, et al: Nutritional-inflammation status and resistance to erythropoietin therapy in haemodialysis patients. Nephrol Dial Transplant 21: 991-998, 2006.

22. Kotanko P, Thijssen S and Levin NW: Association between erythropoietin responsiveness and body composition in dialysis patients. Blood Purif 26: 82-89, 2008. 